\title{
Primary hyperparathyroidism presenting as major depression with psychotic features
}

\section{JosePH KuO 1}

Yone-HaN MAH ${ }^{2,3}$

https://orcid.org/0000-0002-5439-176X

Jui-Teng Wu4

\section{Chin-Chung ShiaO 5,6 \\ https://orcid.org/0000-0003-2220-7574}

${ }^{1}$ Department of Psychiatry, Camillians Saint Mary's Hospital Luodong, Yilan, Taiwan.

2 Division of Gastroenterology, Department of Internal Medicine, Camillians Saint Mary's Hospital Luodong, Yilan, Taiwan.

3Division of Gastroenterology, Department of Internal Medicine, National Taiwan University Hospital and National Taiwan University College of Medicine, Taipei, Taiwan.

${ }^{4}$ Division of General Surgery, Department of Surgery, Camillians Saint Mary's Hospital Luodong, Yilan, Taiwan.

${ }^{5}$ Division of Nephrology, Department of Internal Medicine, Camillians Saint Mary's Hospital Luodong, Yilan, Taiwan.

${ }^{6}$ Saint Mary's Junior College of Medicine, Nursing and Management, Yilan, Taiwan.

Received: 3/5/2019 - Accepted: 5/21/2019

DOl: 10.1590/0101-60830000000219

Kuo J et al. / Arch Clin Psychiatry. 2019;46(6):169-70

\section{Dear Editor,}

Primary hyperparathyroidism (PHPT) might associate with multiple neuropsychiatric symptoms, but it is often overlooked by physicians when making differential diagnosis for patients with psychiatric disorders. We report a case of PHPT presenting as major depression with psychotic features.

A 47-year-old previously healthy woman presented with a 2-weeks history of depressed mood, loss of interest, insomnia, poor concentration, fatigue and poor appetite with prominent body weight loss. Besides, blunted affect, prominent psychomotor retardation, occasional irrelevant speech, the delusion of guilt, persecution, and negation were also noted. Under the impression of major depressive disorder, she was treated with escitalopram
$20 \mathrm{mg}$ and aripiprazole $10 \mathrm{mg}$ per day. After drug treatment for four weeks, the score of the 17-item Hamilton Depression Rating Scale (HAMD-17) was decreased from the initial 46 to 35, which didn't reach obvious response (defined by a decrease of scores for $50 \%$ or more). The following laboratory tests revealed severe hypercalcaemia of 16.8 (reference, 8.6-10.0) $\mathrm{mg} / \mathrm{dl}$, hypokalemia of 2.4 (3.5-5.1) $\mathrm{mg} / \mathrm{dl}$, borderline thyroid function with free-T4 of 1.74 (0.93-1.7) $\mathrm{ng} / \mathrm{dl}$ and thyroid-stimulating hormone of $1.18(0.27-4.2) \mu \mathrm{IU} / \mathrm{ml}$, along with blood urea nitrogen of $26.2(6-20) \mathrm{mg} / \mathrm{dl}$ and creatinine of $1.4(0.5-0.9) \mathrm{mg} / \mathrm{dl}$.

The diagnosis of PHPT was established by the extraordinarily high level of PTH [911.8 (17-65) pg/ml], and one huge mass at left lower parathyroid gland was subsequently found by echography and Tc-99m scan (Figure 1). The parathyroidectomy was undergone soon,

\section{Anterior 25 minutes}

\section{Anterior 4 hours}

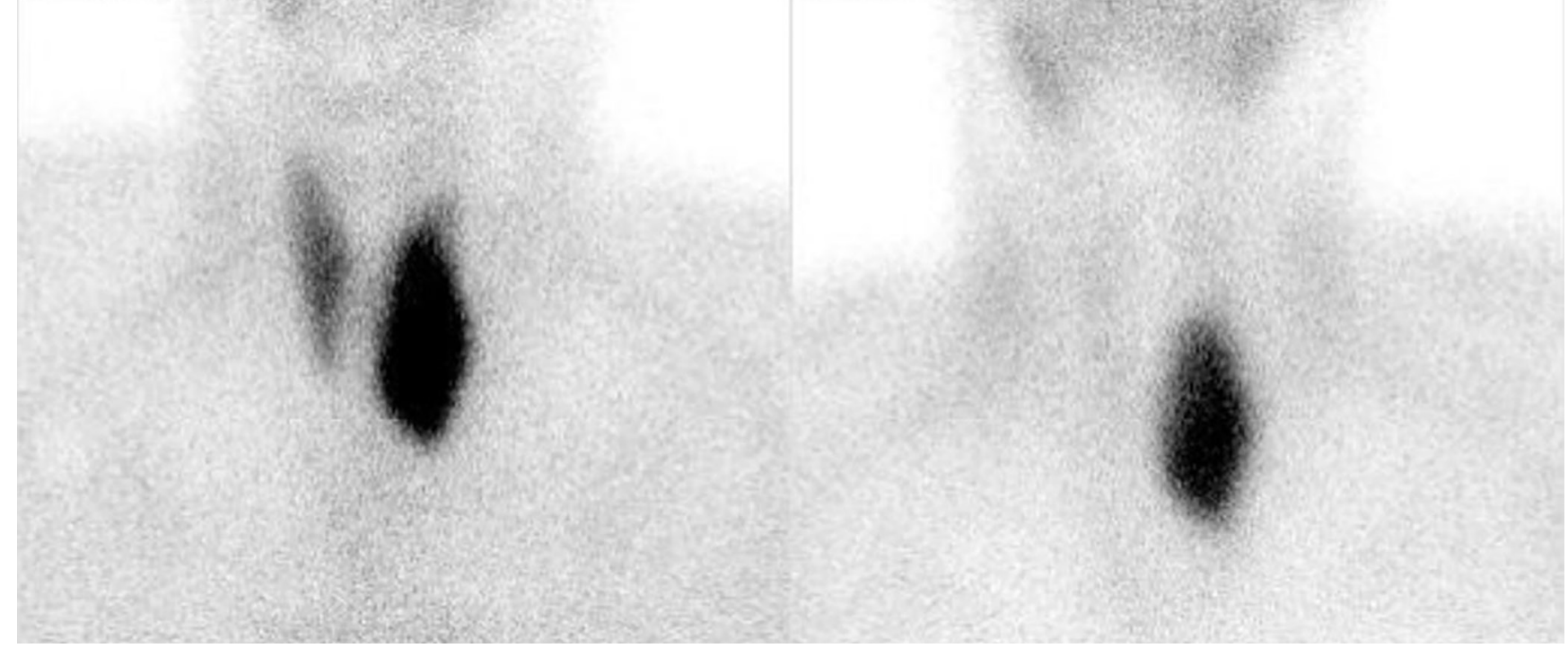

Figure 1. Parathyroid scan with intravenous Technetium-99m methoxyisobutylisonitrile injection revealed a huge parathyroid adenoma in the left lower thyroid region. 
and the pathological report disclosed one $5 \times 3 \times 1.5 \mathrm{~cm}$ parathyroid gland with oncocytic and chief cell hyperplasia. Both serum calcium $(9.9 \mathrm{mg} / \mathrm{dl})$ and PTH levels $(52.57 \mathrm{pg} / \mathrm{ml})$ dramatically decreased to the normal range in one day following the parathyroidectomy. The score of HAMD-17 decreased to 23 in seven days and to 4 in 14 days after the surgery, indicative of complete remission of the depression. Her somatic symptom remitted and her body weight returned back to the same before this episode.

Psychiatric symptoms were reported in $4.2 \%-10 \%$ patients with PHPT ${ }^{1,2}$, whereas higher prevalence rates of neuropsychiatric disturbances were observed in patients with more severe PHPT necessitating parathyroidectomy ${ }^{3}$. These prevalence rates include 43.1\%-53\% for anxiety, $33.0 \%-62.1 \%$ for depression, $22 \%$ for thoughts of death or suicide, $51.9 \%$ for anger and irritability, $5-20 \%$ for hallucination and delusions, and $37.3 \%-46.6 \%$ for impaired cognition 3 . The occurrence and severity of psychiatric symptoms are associated with increased levels of serum calcium and $\mathrm{PTH}^{4}$. The mechanism is presumably related to the low levels of monoamine metabolites and increased calcium levels found in the cerebrospinal fluid 1 .

A primary psychiatric disorder is a diagnosis of exclusion. PHPT should be considered as a differential diagnosis for a patient with a psychiatric disorder. To establish the diagnosis of PHPT, some biochemical tests including serum total calcium, PTH, creatinine, 25-hydroxy vitamin D and 24-hour urinary calcium and creatinine are recommended, and certain disorders causing secondary hyperparathyroidism must be excluded ${ }^{4}$. Parathyroidectomy is the only curative therapy for PHPT, which significantly leads to a reduction of depressive symptoms and an improvement of patients' quality of lives ${ }^{2}$.

\section{Acknowledgment}

The authors declare no potential conflicts of interest in writing this report.

\section{Funding}

None declared.

\section{References}

1. Chiba Y, Satoh K, Ueda S, Kanazawa N, Tamara Y, Horiuchi T. Marked improvement of psychiatric symptoms after parathyroidectomy in elderly primary hyperparathyroidism. Endocr J. 2007;54(3):379-83.

2. Wilhelm SM, Lee J, Printz RA. Major depression due to primary hyperparathyroidism: a frequent and correctable disorder. Am Surg. 2004;70(2):175-9.

3. Parks KA, Parks CG, Onwuameze OE, Shrestha S. Psychiatric complications of primary hyperparathyroidism and mild hypercalcaemia. Am J Psychiatry. 2017;174(7): 620-2.

4. Parnell KE, Oltmann SC. The surgical management of primary hyperparathyroidism: an updated review. Int J Endo Oncol. 2018;5(1):IJE07. 